Check for updates

Cite this: Phys. Chem. Chem. Phys., 2020, 22, 9240

Received 5th March 2020 Accepted 6th April 2020

DOI: $10.1039 / \mathrm{d} 0 \mathrm{cp} 01252 \mathrm{a}$

rsc.li/pccp

\title{
Induced magnetic field in sp-hybridized carbon rings: analysis of double aromaticity and antiaromaticity in cyclo[2N]carbon allotropes $\dagger$
}

\author{
Nickolas D. Charistos (D)*a and Alvaro Muñoz-Castro (D) *b
}

\begin{abstract}
The induced magnetic field of $C_{2 N}(N=3-14)$ carbon rings was dissected to contributions from out-ofplane and in-plane $\pi$ orbitals revealing two concurrent long range shielding or deshielding cones as a manifestation of the dual aromatic and antiaromatic character of $\mathrm{C}_{4 n+2}$ and of $\mathrm{C}_{4 n}$ rings respectively. Aromaticity based on the magnetic criterion was evaluated with regard to the bonding pattern and geometrical characteristics that elucidate the influence of bond length and bond angle alteration on out-of-plane and in-plane magnetic responses. Ground state polyynic geometries of $\mathrm{C}_{4 n+2}$ rings exhibit comparable shielding cones to annulenes, decreasing the magnetic response with regard to the ring size and similar $\pi_{\text {out }}$ and $\pi_{\text {in }}$ diatropicity. Transition state cumulenic rings display increased aromaticity expressed by a very strong constant magnetic response and augmented $\pi_{\text {out }}$ diatropicity with regard to $\pi_{\text {in }}$. The variations of the induced magnetic field are explained on the basis of frontier orbital interactions through rotational excitations, which enable further rationalization of the aromatic/antiaromatic behavior.
\end{abstract}

\section{Introduction}

Since the discovery of buckminsterfullerene $\mathrm{C}_{60}$ in $1985,{ }^{1}$ research on $\mathrm{sp}^{2}$ carbon allotropes such as fullerenes, graphenes ${ }^{2}$ and carbon nanotubes ${ }^{3}$ has sparked the interest on the design and synthesis of a plethora of novel carbon-based nanomaterials with exceptional diversity and applications in numerous fields such as optoelectronics, gas separation, energy conversion and shortage, catalysis and medicine. ${ }^{4-12}$ Around the same time research on different carbon allotropes with mixed $\mathrm{sp}^{2}-\mathrm{sp}$ hybridization $^{13}$ or solely $\mathrm{sp}$ hybridized carbon atoms ${ }^{14}$ has paved the way for the discovery of new all carbon species with promising applications such as graphdiynes ${ }^{15-17}$ and carbon rings. ${ }^{18}$

Early in 1989 Diederich et al. reported the gas phase generation of a highly reactive $\mathrm{C}_{18}$ cyclocarbon $^{14}$ in which each carbon atom was bonded to two other carbons, in contrast to $\mathrm{sp}^{2}$ hybridized allotropes where the bonding pattern involves three-coordinated carbon atoms. The main question that evolved to a controversy by conflicting theoretical studies was on the

\footnotetext{
${ }^{a}$ Aristotle University of Thessaloniki, Department of Chemistry, Laboratory of Quantum and Computational Chemistry, Thessaloniki, 54 124, Greece. E-mail: nicharis@chem.auth.gr

${ }^{b}$ Laboratorio de Química Inorgánica y Materiales Moleculares, Facultad de Ingeniería, Universidad Autonoma de Chile, Llano Subercaceaux 2801, San Miguel, Santiago, Chile. E-mail: alvaro.munoz@uautonoma.cl $\dagger$ Electronic supplementary information (ESI) available. See DOI: 10.1039/ d0cp01252a
}

bonding pattern of $\mathrm{C}_{18}$ : is it polyynic with altering triple and single bonds, or cumulenic with equal bond lengths? Pure and hybrid $\mathrm{DFT}^{19-21}$ as well as MP2 $2^{22,23}$ calculations predict cumulenic structures, whereas $\mathrm{HF},{ }^{14,24}$ quantum Monte Carlo ${ }^{25}$ and $\mathrm{CCSD}^{26}$ calculations predict polyynic geometries. Further studies diagnosed that the DFT calculated bonding pattern depends on the amount of HF exchange of the hybrid functional, ${ }^{27,28}$ whereas range-separated exchange nonempirical DFT schemes provide polyynic structures regardless of the type of functional that is used. ${ }^{29}$ Finally in 2019 Kaiser et al. ${ }^{18}$ isolated by on-surface synthesis and structurally characterized $\mathrm{C}_{18}$ using atomic force microscopy (AFM), revealing a polyynic structure with altering single-triple CC bonds putting an end to the controversy. $^{23,24}$ Moreover, the cumulenic structure has been predicted to be a transition state between two degenerate polyynic ground states with inverted single-triple bonds. ${ }^{25,28,30}$

A unique characteristic of sp-hybridized carbon structures is the presence of two perpendicular $\pi$-electron systems ${ }^{31}$ extending the capabilities from $\mathrm{sp}^{2}$-hybridized carbon structures which sustain a single $\pi$-system. Diederich proposed that $\mathrm{C}_{18}$ benefited from two distinctive sets of delocalized $\pi$ electrons, ${ }^{14}$ one from out-of-plane $\left(\pi_{\text {out }}\right)$ and the other from in-plane $\left(\pi_{\text {in }}\right)$ oriented $18 \pi$ orbitals (Fig. 1), both obeying Huckel's $4 n+2$ rule, which is expected to exhibit interesting electron acceptor properties. ${ }^{27}$ The presence of double aromaticity for $\mathrm{C}_{4 n+2}$ and antiaromaticity for $\mathrm{C}_{4 n}$ cyclocarbons was confirmed via ring current analysis by Fowler et al. ${ }^{19}$ while the large NICS values reported by Suresh ${ }^{20}$ also imply the existence of double aromaticity given by the two 

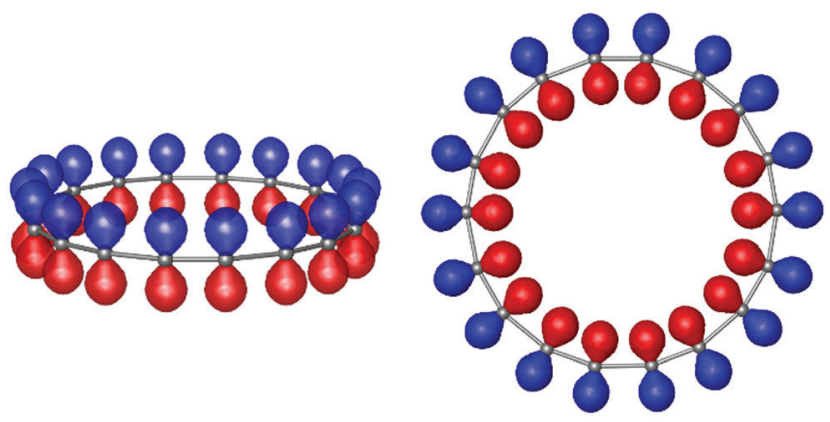

$\pi_{\text {out }}$

$\pi_{\text {in }}$

Fig. 1 Illustration of two orthogonal sets of $p$ orbitals of $C_{18}$ ring displaying perpendicular to the plane $\left(\pi_{\text {out }}\right)$ and in-plane $\left(\pi_{\text {in }}\right)$ orientations.

perpendicular $\pi$-systems. The double aromaticity of small carbon rings and related boron-carbon molecules was also confirmed with the CMO-NICS analysis put forth by Schleyer et $a l^{32}$ However, studies on the aromaticity of carbon rings were performed on cumulenic structures and the effect of bonding pattern on the magnetic response was not evaluated. Recently Baryshnikov et al. ${ }^{28}$ employed the GIMIC method to measure the ring currents of both polyynic and cumulenic configurations of $\mathrm{C}_{18}$ and reported that the polyynic structure induces a strong diatropic current which is however much weaker than the diatropic current of cumulene. Moreover, although the GIMIC method does not yet allow the dissection of ring current to contributions from different sets of orbitals, it obtained the current strengths of $\pi_{\text {out }}$ and $\pi_{\text {in }}$ sets by integrating the current density within distinctive contours and found that $\pi_{\text {out }}$ orbitals induce stronger diatropic currents than $\pi_{\mathrm{in}}$, both in polyynic and cumulenic geometries.

Herein, we study the distinctive characteristics of double aromaticity and antiaromaticity based on the magnetic criterion ${ }^{33}$ from cyclo[ $2 N]$ carbon allotropes, given by their peculiar perpendicular $\pi$-systems. However we note that a thorough investigation of the aromaticity requires to take into consideration both electronic and energetic criteria. ${ }^{34-36}$ We perform a detailed analysis of the magnetic response of $\mathrm{C}_{2 N}(N=3-14)$ rings which is directly related to the aromatic/antiaromatic behavior, ${ }^{3-42}$ by means of canonical orbital contributions to the induced magnetic field (CMO-IMF) that allows the through-space visualization of shielding and deshielding cones induced by different sets of orbitals, as well as from each CMO. ${ }^{43-46}$ Hence the magnetic field induced from each $\pi_{\text {out }}$ and $\pi_{\text {in }}$ set was visualized for polyynic and cumulenic geometries of $\mathrm{C}_{4 n+2}$ rings, as well as for those of $\mathrm{C}_{4 n}$ rings, revealing the trends of the magnetic response of each set of MOs and the effect of ring size, bond length and bond angle. Our results show that polyynic geometries induce a cumulative magnetic field from both sets which is comparable to annulenes and declines with the evolution of ring size, whereas cumulenes induce a much stronger magnetic field independent of the ring size. Accordingly, $\mathrm{C}_{4 n}$ rings display decreasing deshielding cones for both sets with regard to the ring size. These trends are associated with the inherent bonding pattern and geometrical characteristics of carbon rings, which are further explained on the basis of frontier orbital interactions through rotational excitations.

\section{Computational methods}

A number of various exchange-correlation functionals (XCs) were tested for the optimization of $\mathrm{C}_{18}$ (see ESI, $\dagger$ Table S1). Functionals with a high percentage of HF exchange (HFX > $50 \%$ ) yielded polyynic structures, whereas pure GGAs and functionals with a low percentage of HFX resulted in cumulenic structures with no bond length alteration (BLA) (Table S1, $\mathrm{ESI} \dagger$ ). In this work we have chosen the use of the rangeseparated hybrid $\omega \mathrm{B} 97 \mathrm{XD}^{47}$ for the optimization of the $\mathrm{C}_{2 N}$ $(N=3-14)$ molecules because it correctly reproduces the experimentally realized ${ }^{18}$ polyynic geometry of $\mathrm{C}_{18}$ and it has been reported to have the best performance among other functionals for the description of polyynic structures. ${ }^{48}$ Moreover, long-range corrected XC functionals like $\omega$ B97XD have been found to provide a better description of the cyclic electron delocalization in monocyclic and polycyclic aromatics with regard to conventional XC functionals. ${ }^{36}$ All geometry optimizations were carried out with Gaussian $09^{49}$ using the 6-311++G(d,p) Gaussian basis set.

Nucleus independent chemical shieldings (NICS) were calculated within the GIAO formalism using several functionals, as detailed in ESI, $\dagger$ and the triple- $\zeta$ slater type basis set with two polarization functions (TZ2P) employing the ADF2019 software. $^{50,51}$ BHandHLYP $^{52}$ was chosen for the quantitative analysis of dissected NICS $_{z z}$ because it performs in very good agreement with $\omega \mathrm{B} 97 \mathrm{XD}$ (Table S2 and Fig. S2, ESI $\dagger$ ) and it is available for the CMO analysis of the chemical shift in ADF. The computationally affordable $\mathrm{PBE}^{53}$ was chosen for the qualitative analysis of the induced magnetic field. Although the PBE was found to overestimate the NICS, especially in polyynic structures, it accurately reproduces the qualitative features of the magnetic response with regard to the ring size and bonding pattern of $\mathrm{C}_{2 N}$ rings (see ESI, $\dagger$ Fig. S2 and S3). Chemical shieldings were dissected to contributions from canonical MOs (CMOs) employing the NMR and EPR modules ${ }^{54}$ of the ADF and the contributions of $\pi_{\text {out }}$ and $\pi_{\text {in }}$ sets of orbitals were constructed by the summation of the corresponding CMOs.

For the calculation of the induced magnetic field the molecules were placed on $x y$ Cartesian plane and the chemical shielding was calculated in a $31^{3}$ cubic grid of points with a separation step of $0.5 \AA$. Extraction of CMO contributions at each point and generation of cube files per MO were performed with our custom MIMAF code. ${ }^{55}$ Visualization of induced magnetic field isosurfaces was performed with the VMD. ${ }^{56}$

\section{Results and discussion}

\section{Geometrical characteristics}

The ground state optimized geometries of $\mathrm{C}_{4 n+2}(n=1-6)$ and $\mathrm{C}_{4 n}(n=2-6)$ molecules are shown in Fig. S1 (ESI $\left.\dagger\right)$ and their corresponding geometrical parameters are given in Table 1. 
Table 1 Geometrical parameters of $C_{2 N}(N=3-14)$ rings optimized at the $\omega B 97 X D / 6-311++G(d, p)$ level

\begin{tabular}{llllllrr}
\hline Molecule & Point Group & $d_{1}(\AA)$ & $d_{2}(\AA)$ & BLA $^{a}$ & $\theta_{1}\left({ }^{\circ}\right)$ & $\theta_{2}\left({ }^{\circ}\right)$ & BAA $^{b}$ \\
\hline $\mathrm{C}_{6}$ & $D_{3 \mathrm{~h}}$ & 1.321 & & 0.00 & 149.2 & 90.8 & 58.4 \\
$\mathrm{C}_{8}$ & $C_{4 \mathrm{~h}}$ & 1.384 & 1.251 & 0.133 & 162.8 & 107.2 & 55.6 \\
$\mathrm{C}_{10}$ & $D_{5 \mathrm{~h}}$ & 1.290 & 1.290 & 0.00 & 165.0 & 123.0 & 42.1 \\
$\mathrm{C}_{12}$ & $C_{6 \mathrm{~h}}$ & 1.359 & 1.234 & 0.126 & 168.3 & 131.7 & 36.6 \\
$\mathrm{C}_{14}$ & $C_{7 \mathrm{~h}}$ & 1.307 & 1.256 & 0.051 & 167.0 & 141.6 & 25.4 \\
$\mathrm{C}_{14} \mathrm{TS}$ & $D_{7 \mathrm{~h}}$ & 1.281 & & 0.00 & 168.1 & 140.5 & 27.6 \\
$\mathrm{C}_{16}$ & $C_{8 \mathrm{~h}}$ & 1.364 & 1.216 & 0.148 & 158.9 & 156.1 & 2.8 \\
$\mathrm{C}_{18}$ & $C_{9 \mathrm{~h}}$ & 1.346 & 1.223 & 0.123 & 160.2 & 159.8 & 0.4 \\
$\mathrm{C}_{18} \mathrm{TS}$ & $D_{9 \mathrm{~h}}$ & 1.277 & & 0.00 & 169.4 & 150.6 & 18.8 \\
$\mathrm{C}_{20}$ & $C_{10 \mathrm{~h}}$ & 1.358 & 1.216 & 0.141 & 162.6 & 161.4 & 1.2 \\
$\mathrm{C}_{22}$ & $C_{11 \mathrm{~h}}$ & 1.351 & 1.219 & 0.132 & 164.5 & 162.8 & 1.7 \\
$\mathrm{C}_{22} \mathrm{TS}$ & $D_{11 \mathrm{~h}}$ & 1.276 & & 0.00 & 171.0 & 156.3 & 14.8 \\
$\mathrm{C}_{24}$ & $C_{12 \mathrm{~h}}$ & 1.355 & 1.216 & 0.139 & 165.8 & 164.2 & 1.7 \\
$\mathrm{C}_{26}$ & $C_{13 \mathrm{~h}}$ & 1.352 & 1.217 & 0.135 & 167.0 & 165.3 & 1.7 \\
$\mathrm{C}_{26} \mathrm{TS}$ & $D_{13 \mathrm{~h}}$ & 1.276 & & 0.00 & 172.7 & 159.6 & 13.1 \\
$\mathrm{C}_{28}$ & $C_{14 h}$ & 1.354 & 1.216 & 0.138 & 168.0 & 166.3 & 1.7
\end{tabular}

${ }^{a}$ Bond length alteration, $d_{1}-d_{2} \cdot{ }^{b}$ Bond angle alteration, $\theta_{1}-\theta_{2}$.

All molecules adopt $C_{(n / 2) \mathrm{h}}$ point group symmetry representing polyynic structures with altering bond lengths and altering bond angles, except for $\mathrm{C}_{6}$ and $\mathrm{C}_{10}$ which adopt $D_{(n / 2) \mathrm{h}}$ cumulenic structures with equal bond lengths and altering angles. The bond length alteration (BLA) ranges from $0.123 \AA$ to $0.148 \AA$ for all $C_{(n / 2) \mathrm{h}}$ point group molecules, except for $\mathrm{C}_{14}$ which presents a minimal BLA of 0.051 A. On the other hand, bond angle alteration (BAA) is very large for small rings starting from $58.4^{\circ}$ and $55.6^{\circ}$ for $\mathrm{C}_{6}$ and $\mathrm{C}_{8}$, respectively, and decreases rapidly as the ring size increases, reaching a constant value of $1.7^{\circ}$ for $\mathrm{C}_{2 N}(N=11-14)$, while the smallest BAA $\left(0.4^{\circ}\right)$ is observed in $\mathrm{C}_{18}$. For $\mathrm{C}_{4 n}$ molecules $D_{(n / 2) \mathrm{h}}$ geometries with altering bond lengths and equal bond angles were found to be first-order saddle points, whereas higher symmetry $D_{n h}$ geometries with equal bonds and angles were found to be higher-order saddle points for both $\mathrm{C}_{4 n+2}$ and $\mathrm{C}_{4 n}$ molecules.

For $\mathrm{C}_{4 n+2}(n=3-6)$ molecules, QST2 and IRC calculations generated cumulenic geometries with equal bond lengths and altering bond angles, adopting $D_{(n / 2) \mathrm{h}}$ point group symmetry, as transition states between two inversion related polyynic ground states $^{28}$ (Fig. 2). The transition states display increased BAA with regard to the respective ground states. The energy barrier for the inversion increases with the number of carbon atoms, starting from a marginal barrier of $0.12 \mathrm{kcal} \mathrm{mol}^{-1}$ for $\mathrm{C}_{14}$ and reaching 10.90, 24.42 and $37.54 \mathrm{kcal} \mathrm{mol}^{-1}$ for $\mathrm{C}_{18}, \mathrm{C}_{22}$ and $\mathrm{C}_{26}$ respectively.

\section{Total magnetic response}

When an (anti)aromatic molecule is subjected to a uniform external magnetic field $\vec{B}^{\text {ext }}$, then closed circuits of mobile electrons induce a current density, which in turn induces a secondary magnetic field, $\vec{B}^{\text {ind }}$, either opposing (shielding) the external field in aromatic rings or reinforcing (deshielding) the external field in antiaromatic rings. In planar structures the molecule is oriented such that the molecular plane $(x y)$ is perpendicular to the external field's direction $(z)$, and hence the induced magnetic field is designated as $\vec{B}_{z}^{\text {ind }}$. The induced

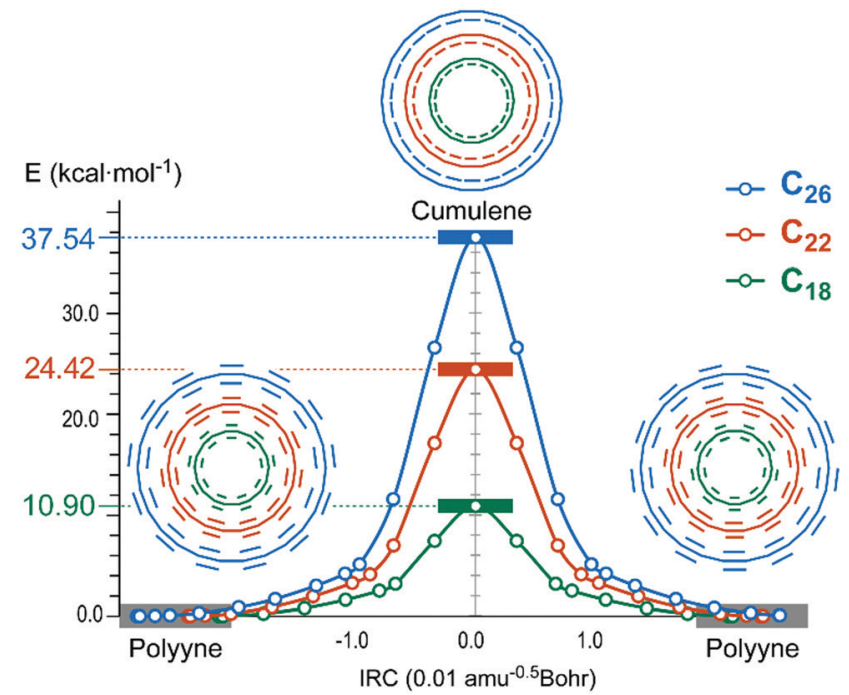

Fig. 2 Relative IRC profiles of $C_{18}, C_{22}$ and $C_{26}$ depicting the interchange of single-triple bonds of polyynic ground states through a cumulenic transition state.

magnetic field is typically visualized as isosurfaces of $B_{z}^{\text {ind }}$, forming long range shielding or deshielding cones in aromatic or antiaromatic rings respectively. The value of $B_{z}^{\text {ind }}$ isosurface at any point is equal to the $z z$ tensor component of nucleus independent chemical shift, $\mathrm{NICS}_{z z}$.

NICS $_{z z}$ values, presented in Table 2 and Fig. S2 (ESI $\dagger$ ), reveal the characteristic features of carbon rings' magnetic response. These features are as follows: (a) $\mathrm{C}_{4 n+2}$ molecules display large diatropic (negative) NICS $_{z z}$ representative of aromatic character, whereas $\mathrm{C}_{4 n}$ molecules display large paratropic (positive) $\mathrm{NICS}_{z z}$ representative of antiaromatic character; (b) NICS $_{z z}$ of polyynic $\mathrm{C}_{4 n+2}(n=3-6)$ and $\mathrm{C}_{4 n}$ molecules decreases significantly as the ring size increases; (c) $\operatorname{NICS}_{z z}$ of the cumulenic $\mathrm{C}_{4 n+2}(n=1-6)$ geometries increases with the ring size, showing a trend to reach a maximum value at larger rings; (d) accordingly for $\mathrm{C}_{4 n+2}$ molecules there is an increasing difference in $\operatorname{NICS}_{z z}$ between polyynic ground states and cumulenic transition states with an increment in the ring size; (e) the maximum $\operatorname{NICS}_{z z}$ among

Table 2 Total NICS zalues (ppm) and contributions from $\pi_{\text {out }}$ and $\pi_{\text {in }}$ orbitals of $\mathrm{C}_{2 N}$ carbon rings and reference molecules computed at the BHandHLYP/TZ2P level

\begin{tabular}{lrrrlrrr}
\hline Molecule & \multicolumn{1}{c}{ Total } & \multicolumn{1}{l}{$\pi_{\text {out }}$} & \multicolumn{1}{l}{$\pi_{\text {in }}$} & Molecule & Total & $\pi_{\text {out }}$ & $\pi_{\text {in }}$ \\
\hline $\mathrm{C}_{6}$ & -37.7 & -39.8 & 19.2 & $\mathrm{C}_{8}$ & 130.9 & 110.5 & 28.2 \\
$\mathrm{C}_{10}$ & -65.2 & -52.1 & -3.3 & $\mathrm{C}_{12}$ & 96.8 & 64.8 & 34.1 \\
$\mathrm{C}_{14}$ & -81.6 & -49.9 & -25.4 & $\mathrm{C}_{16}$ & 57.9 & 27.0 & 31.7 \\
$\mathrm{C}_{14}$ TS & -89.2 & -56.7 & -25.0 & $\mathrm{C}_{20}$ & 33.4 & 15.5 & 18.7 \\
$\mathrm{C}_{18}$ & -44.7 & -20.6 & -16.4 & $\mathrm{C}_{24}$ & 18.7 & 8.6 & 10.8 \\
$\mathrm{C}_{18}$ TS & -101.5 & -58.3 & -37.2 & $\mathrm{C}_{28}$ & 10.5 & 4.8 & 6.4 \\
$\mathrm{C}_{22}$ & -22.0 & -10.7 & -7.8 & $\mathrm{C}_{8} \mathrm{H}_{8}$ & 107.2 & 90.2 & \\
$\mathrm{C}_{22}$ TS & -104.0 & -58.1 & -40.7 & & & & \\
$\mathrm{C}_{26}$ & -10.9 & -5.4 & -3.4 & & & & \\
$\mathrm{C}_{26}$ TS & -99.3 & -55.8 & -39.0 & & & & \\
$\mathrm{C}_{6} \mathrm{H}_{6}$ & -15.9 & -36.7 & & & & & \\
$\mathrm{C}_{18} \mathrm{H}_{18}$ & -41.5 & -48.0 & & & & & \\
$\mathrm{C}_{18} \mathrm{H}_{6}$ & -8.1 & -12.7 & -15.7 & & & & \\
$\mathrm{~B}_{9} \mathrm{H}_{9}$ & 3.3 & 0.5 & 3.4 & & & &
\end{tabular}


ground state $\mathrm{C}_{4 n+2}$ molecules is observed in $\mathrm{C}_{14}$ which presents the minimum BLA.

The above remarks clarify controverting NICS studies of $\mathrm{C}_{4 n+2}$ rings. ${ }^{19,20}$ The NICS values reported by Fowler et al. show an increment in diatropic NICS of $\mathrm{C}_{4 n+2}$ for $n=1-5$ and then a significant decrement for $n=6,7$ due to molecular geometry obtained with B3LYP functional which predicts cumulenic structures for $n=1-5$ and polyynic structures for $n=6,7 .{ }^{19}$ On the other hand, Suresh and Remya reported increasing diatropic NICS values for all $\mathrm{C}_{4 n+2}(n=1-7)$ trending to a maximum value due to cumulenic geometries obtained with MO6L functional. ${ }^{20}$

Visualizations of $B_{z}^{\text {ind }}$ induced by all electrons of $C_{2 N}$ molecules are presented in Fig. 3 using multiple clipped isosurfaces ( $\pm 5 \mathrm{ppm}$ and $\pm 20 \mathrm{ppm}$ ) to depict the long-range effect, as well as a full isosurface of large $B_{z}^{\text {ind }}$ values to illustrate specific characteristics of the magnetic response close to the molecular domain. $\mathrm{C}_{4 n+2}$ molecules induce strong long-range shielding cones representative of strong aromatic character. The longrange shielding cone (cyan, $-5 \mathrm{ppm}$ ) of $\mathrm{C}_{4 n+2}$ molecules increases with the ring size but the actual trend is revealed by inspection of the strong response close to the molecular plane depicted with green isosurfaces $(-50 \mathrm{ppm})$ in Fig. 3. In ground state polyynic structures the extension of the short-range response increases from $\mathrm{C}_{10}$ to $\mathrm{C}_{14}$ where it reaches a maximum span forming a uniform shielding cone, and then gradually decreases from $\mathrm{C}_{18}$ to $\mathrm{C}_{26}$, deforming to a toroidal shape inside the ring of $\mathrm{C}_{26}$. On the contrary the strong shielding cone of cumulenic transition states retains its uniform long range shape. Therefore, the weakening of the magnetic response in ground states is attributable to polyynic bonding and is irrelevant to the ring size. However, the discrepancy in the magnetic response between ground and transition states for $\mathrm{C}_{18}, \mathrm{C}_{22}$ and $\mathrm{C}_{26}$ is apparently evolving with the ring size. Thus, according to the magnetic criterion, the aromaticity of polyynic $\mathrm{C}_{4 n+2}$ decreases for $n>3$ while it is retained for the respective cumulenic transition states.

$\mathrm{C}_{4 n}$ molecules sustain strong long-range deshielding cones representative of strong antiaromatic character. However strong short-range response $(+60 \mathrm{ppm})$ decreases with the evolution of ring size, starting with extended uniform deshielding cones in $\mathrm{C}_{8}$ and $\mathrm{C}_{12}$ and downgrading to a toroidal shape in $\mathrm{C}_{24}$, which implies the weakening of antiaromaticity.

\section{Magnetic responses of $\pi_{\text {out }}$ and $\pi_{\text {in }}$ orbitals}

The total magnetic response suggests the (anti)aromatic character of $\mathrm{C}_{2 N}$ carbon rings but does not give any clear information about the source of the long range (de)shielding cones and the individual role of each (para)diatropic circuit in the double aromatic character, because it is constructed by contributions from all $\pi_{\text {out }}$,
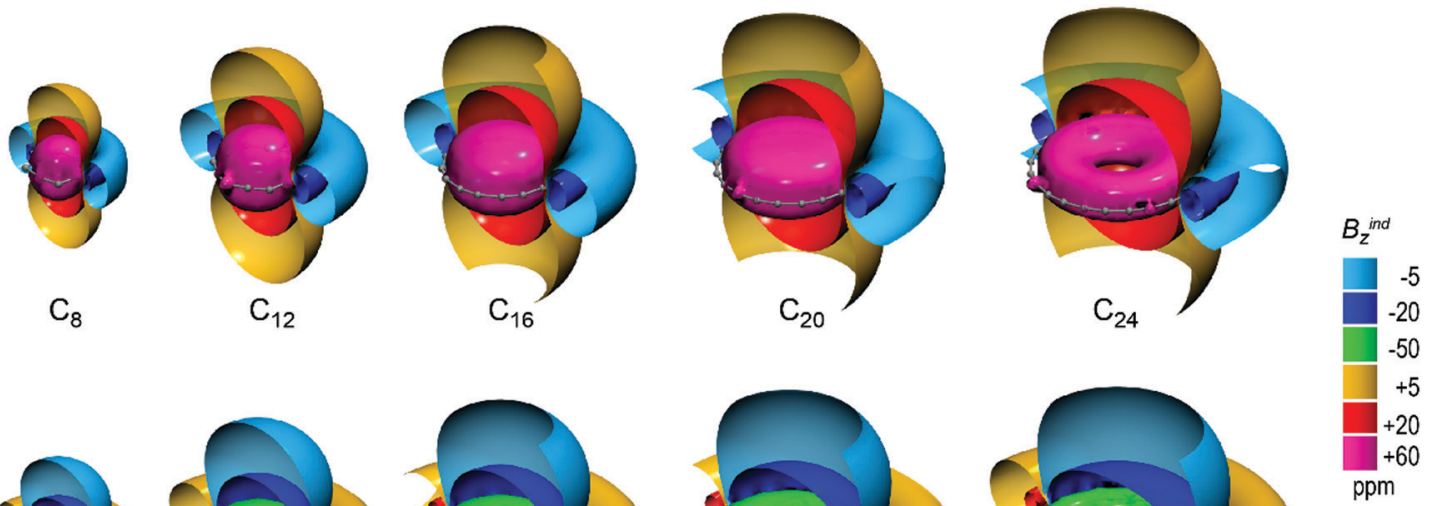

GS
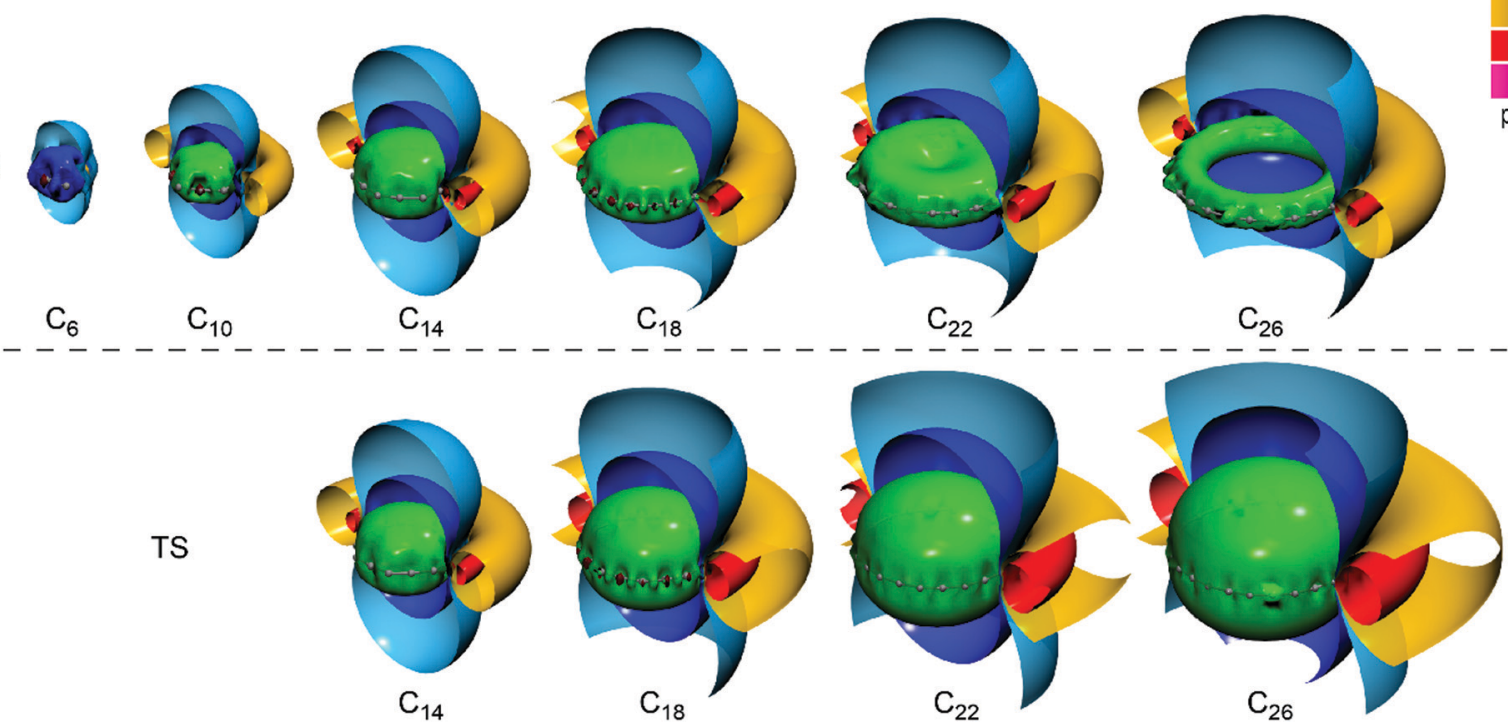

Fig. 3 Isosurfaces of total $B_{z}^{\text {ind }}$ of transition states $D_{(n / 2) h} C_{4 n+2}(n=3-6)$ (bottom row), ground states $C_{(n / 2) h} C_{4 n+2}(n=1-6)(m i d d l e ~ r o w)$ and $C_{4 n}(n=$ 2-6) (top row). 
$\pi_{\text {in }}, \sigma$ and core orbitals. In order to gain insight into the origins and the duality of the (anti)aromatic character of $\mathrm{C}_{2 N}$ carbon rings, we dissected the magnetic response to contributions from $\pi_{\text {out }}$ and $\pi_{\text {in }}$ sets of orbitals, which are exclusive sources of the strong long-range magnetic response.

In Fig. 4, the respective magnetic responses of $\pi_{\text {out }}$ and $\pi_{\text {in }}$ sets of orbitals of ground states $\mathrm{C}_{4 n+2}$ molecules are given. It is clear that for $\mathrm{C}_{14}$ and larger rings, both $\pi_{\text {out }}$ and $\pi_{\text {in }}$ orbitals induce long range shielding cones, illustrating the dual source of their diatropicity. Smaller members $\mathrm{C}_{6}$ and $\mathrm{C}_{10}$ display no or a very weak in-plane magnetic response, respectively, due to a large bond angle alteration (BAA) which leads to a small inplane orbital overlap. Specifically, the $\pi_{\text {out }}$ orbitals of $\mathrm{C}_{6}$ induce a shielding cone very similar to benzene and have comparable $\pi_{\text {out }}-\mathrm{NICS}_{z z}$ values $\left(-39.8\right.$ and $-36.7 \mathrm{ppm}$ for $\mathrm{C}_{6}$ and $\mathrm{C}_{6} \mathrm{H}_{6}$ respectively). The $\pi_{\text {in }}$ orbitals of $\mathrm{C}_{6}$ induce a weak short range diatropic response with a paratropic sphere inside the ring $(+19.2 \mathrm{ppm}$ at ring center), which is representative of localized CC $\sigma$ bonding. ${ }^{43}$ Therefore, $\mathrm{C}_{6}$ displays equal diatropicity with benzene originating from the out-of-plane $\pi$ system, and the large difference in their total $\operatorname{NICS}_{z z}$ does not arise from delocalized electrons but from localized $\sigma$ bonds which have larger paratropic contributions in benzene. $\mathrm{C}_{10}$ presents a strong long-range shielding cone induced from $\pi_{\text {out }}$ orbitals with $-52.1 \mathrm{ppm}$ contributions at the ring center, and a considerably weaker shielding cone induced from $\pi_{\text {in }}$ orbitals with only $-3.3 \mathrm{ppm}$ at the ring center, denoting a marginal in-plane $\pi$ magnetic response due to a large BAA.

$\mathrm{C}_{14}$ induces long range shielding cones from both $\pi_{\text {out }}$ and $\pi_{\text {in }}$ orbitals but the out-of-plane response is much stronger than that of the in-plane, as $\pi_{\text {out }}$ forms an extended uniform $-40 \mathrm{ppm}$ isosurface, while $\pi_{\text {in }}$ forms a condensed shielding toroid inside the ring and the $\pi_{\text {out }}-\mathrm{NICS}_{z z}(-49.9 \mathrm{ppm})$ is the double of $\pi_{\mathrm{in}}-\mathrm{NICS}_{z z}(-25.4 \mathrm{ppm})$. This difference is attributed to the geometry of $\mathrm{C}_{14}$, which presents the minimum BLA that favors the out-of-plane delocalization and significant BAA that hinders the in-plane delocalization. The corresponding transition state displays an almost identical magnetic response, both in terms of shielding cones and $\mathrm{NICS}_{z z}$ values, due to small structural differences in the two states.

For the ground states of larger rings $\mathrm{C}_{18}, \mathrm{C}_{22}$ and $\mathrm{C}_{26}, \pi_{\text {out }}$ and $\pi_{\text {in }}$ display very similar shielding cones. The $\pi_{\text {in }}-\mathrm{NICS}_{z z}$ is only 2-4 ppm less diatropic than $\pi_{\text {out }}-\mathrm{NICS}_{z z}$, denoting a marginally increased out-of-plane magnetic response. The diatropicity decreases with an increment in the ring size for both sets of orbitals. Specifically $\operatorname{NICS}_{z z}$ of $\pi_{\text {out }}\left(\pi_{\text {in }}\right)$ decrease from $-20.6(-16.4)$ ppm in $\mathrm{C}_{18}$ down to $-10.7(-7.8)$ and $-5.4(-3.4)$ ppm in $\mathrm{C}_{22}$ and $\mathrm{C}_{26}$ respectively.

In order to assess the $\mathrm{C}_{18}$ diatropicity we used as reference three prototypical molecules with $18-\pi$ electrons and varying degrees of aromaticity, namely 18-annulene, 18-dehydroannulene and the $\mathrm{BN}$ analogue ${ }^{57} \mathrm{~B}_{9} \mathrm{~N}_{9}$, as presented in Fig. 5. Compared to the $\pi$ response of 18 -annulene (Fig. $5 \mathrm{~b}$ ), the $\pi_{\text {out }}$ of $\mathrm{C}_{18}$ induces a weaker shielding cone and presents less than half $\operatorname{NICS}_{z z}$, denoting a considerable weaker out-of-plane magnetic response in $\mathrm{C}_{18}$. If we consider both $\pi_{\text {out }}$ and $\pi_{\text {in }}$ orbitals of $\mathrm{C}_{18}$, their cumulative shielding cone (Fig. 5a) is greater than $B_{\pi z}^{\text {ind }}$ of 18-annulene, but this is due to the overestimation of PBE which predicts a total $\left(\pi_{\mathrm{out}}+\pi_{\mathrm{in}}\right)-\mathrm{NICS}_{z z}$ value $21 \mathrm{ppm}$ to be more diatropic than NICS $\pi z z$ of $\mathrm{C}_{18} \mathrm{H}_{18}$, while with BHandHLYP it is 11.0 ppm less diatropic. Therefore, $\mathrm{C}_{18}$ displays a similar or a slightly weaker diatropicity when compared to $\mathrm{C}_{18} \mathrm{H}_{18}$. Furthermore, 18-dehydroannule (Fig. 5c) is less aromatic than $\mathrm{C}_{18}$ and induces a weaker $\pi_{\text {out }}$ shielding cone $\left(\pi_{\text {out }}-\mathrm{NICS}_{z z}\right.$ is $\left.-12.7 \mathrm{ppm}\right)$, as well as a weak short-range paratropic response from $12 \pi_{\text {in }}$ electrons $\left(\pi_{\mathrm{in}}-\mathrm{NICS}_{z z}\right.$ is $+15.7 \mathrm{ppm}$ ). Finally, $\mathrm{B}_{9} \mathrm{~N}_{9}$ (Fig. $5 \mathrm{~d}$ ) is a characteristic example with extreme localized electrons, with both $18 \pi_{\text {out }}$ and $18 \pi_{\text {in }}$ electrons inducing weak short range diatropic cones strictly positioned on nitrogen atoms. Hence $\mathrm{C}_{4 n+2}(n>3)$ carbon rings, although they exhibit dual source of diatropicity, show that their magnetic responses are comparable to those of classical $\pi$-aromatic annulenes.

The magnetic responses of transition states, presented in Fig. 6, display constant strong shielding cones of both $\pi_{\text {out }}$ and $\pi_{\mathrm{in}}$. Specifically, $\pi_{\mathrm{out}}-\mathrm{NICS}_{z z}$ retains a value of around $-57 \mathrm{ppm}$, whereas $\pi_{\mathrm{in}}-\mathrm{NICS}_{z z}$ amounts to $\sim-39 \mathrm{ppm}$ for all transition

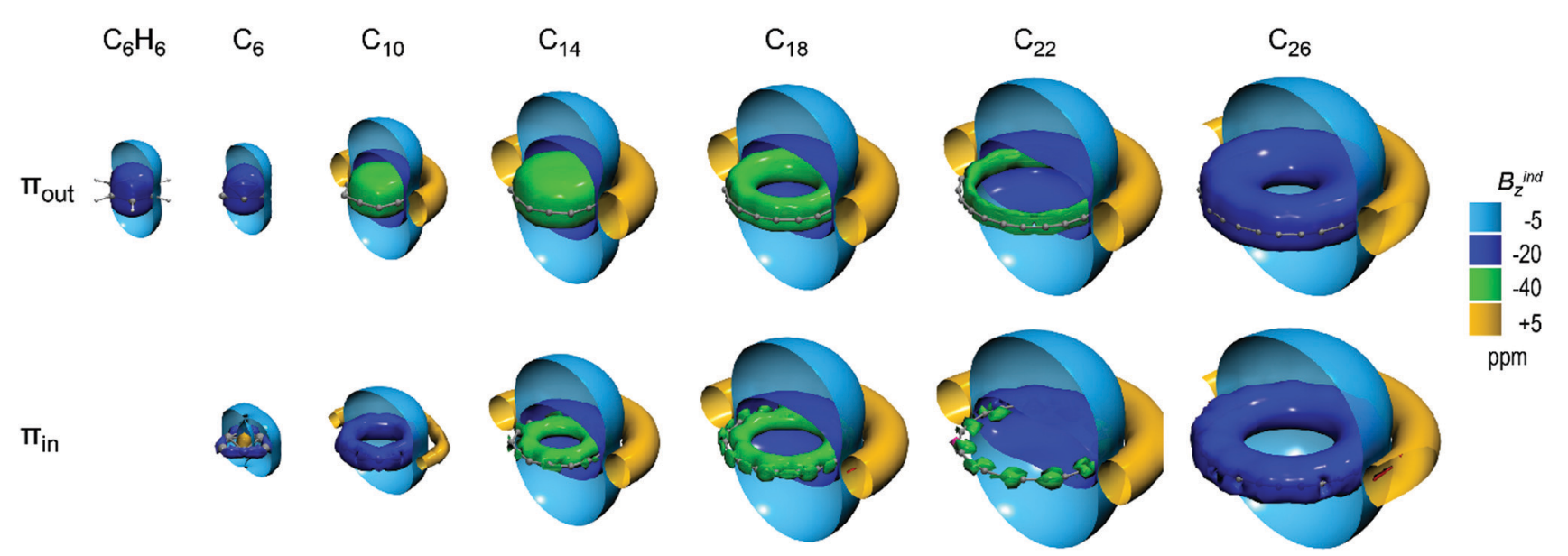

Fig. 4 Isosurfaces of $\pi_{\text {out }}$ (top) and $\pi_{\text {in }}$ (bottom) contributions to $B_{z}^{\text {ind }}$ of polyynic ground states of $C_{(n / 2) h} C_{4 n+2}(n=1-6)$ rings. 
(a)
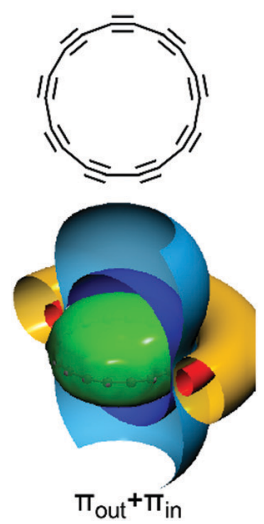

(b)
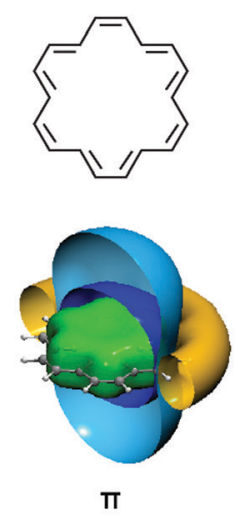

(c)
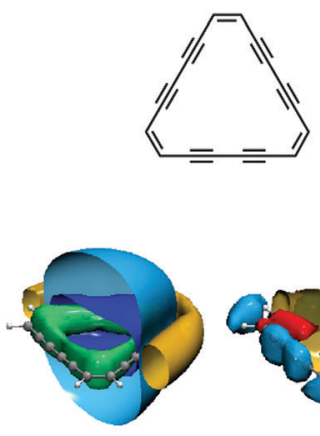

$\pi_{\text {out }}$

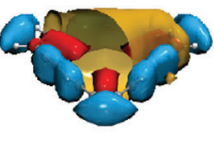

$\pi_{\text {in }}$ (d)
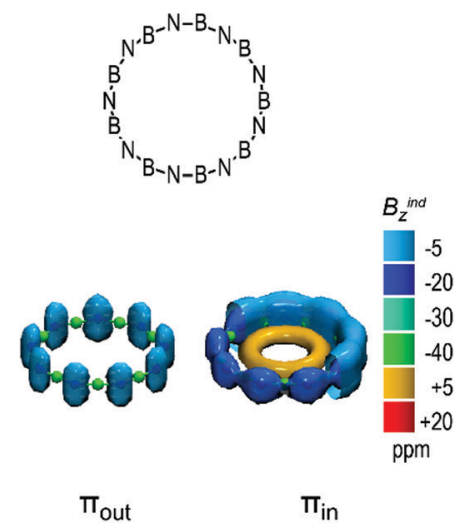

Fig. 5 Isosurfaces of $\pi$ contributions to $B_{z}^{\text {ind }}$ of (a) $C_{9 h} C_{18}$, (b) 18-annulene, (c) 18-dehydroannulene and (d) $B_{9} N_{9}$.

states, implying favorable $\pi_{\text {out }}$ delocalization. The difference in $\pi_{\text {out }}$ and $\pi_{\text {in }}$ can be explained by the increased BAA of cumulenic structures which hinders the in-plane overlap. Indeed, the third-order saddle point $D_{18 \mathrm{~h}}$ geometry of $\mathrm{C}_{18}$ with zero BLA and BAA presents almost equal $\pi_{\text {out }}$ and $\pi_{\text {in }}$ NICS $_{z z}$ values ( -60.2 and $-58.7 \mathrm{ppm}$ respectively). However, $\pi_{\text {in }}$ electrons in cumulenic geometries are still more diatropic than polyynic. Hence BLA is the main factor that constrains both $\pi_{\text {out }}$ and $\pi_{\text {in }}$ magnetic responses and BAA affects secondarily only the $\pi_{\text {in }}$ magnetic response. Consequently, the cumulenic geometries are very diatropic from both sources of magnetic response.

Concerning the antiaromatic $\mathrm{C}_{4 n}(n=2-6)$ rings, both $\pi_{\text {out }}$ and $\pi_{\text {in }}$ orbitals induce equivalent long range deshielding cones (Fig. 7), except for $\mathrm{C}_{8}$ which exhibits only $\pi_{\text {out }}$ deshielding cone and a weak short range paratropic response of $\pi_{\text {in }}$ due to large BAA, which does not justify a paratropic in-plane ring current. The $\pi_{\text {in }}$ response of $\mathrm{C}_{14}$ is also weak due to large BAA but still forms a deshielding cone denoting weak in-plane paratropic current, whereas for larger rings $\pi_{\text {out }}$ and $\pi_{\text {in }}$ induce equivalent deshielding cones. The $\pi_{\text {out }}$ deshielding cones of $\mathrm{C}_{8}$ and $\mathrm{C}_{12}$ are comparable to that of planar $\mathrm{C}_{8} \mathrm{H}_{8}$ but decline significantly for larger rings, as the $\pi_{\text {in }}$ deshielding cones also do. Hence, $\mathrm{C}_{4 n}$ $(n=3-6)$ rings exhibit a dual source of paratropicity representative of antiaromatic character which weakens significantly with an increment in the ring size.

\section{CMO contributions}

In order to elucidate the above remarks, we have to take into consideration the electronic structure of carbon rings, especially the frontier orbitals that principally determine the overall magnetic response. Dissection of the induced magnetic field to CMO contributions reveals that lower energy valence orbitals induce a diatropic response, whereas the highest occupied orbitals dictate the overall magnetic response. ${ }^{43,44}$ Indeed in $\mathrm{C}_{2 N}$, the contributions of $\pi_{\text {out }}$ and $\pi_{\text {in }}$ to NICS $_{z z}$ are linearly correlated to the contribution of the corresponding HOMOs $\left(R^{2}=0.97\right.$, Fig. S4, ESI $\left.\dagger\right)$. Hence the (para)diatropicity can be analyzed in terms of HOMO contributions, as given in Table S4 and Fig. S5, S6 (ESI $\dagger$ ).

Generally, in antiaromatic molecules the HOMOs induce a very strong paratropic response that overwhelms the diatropicity

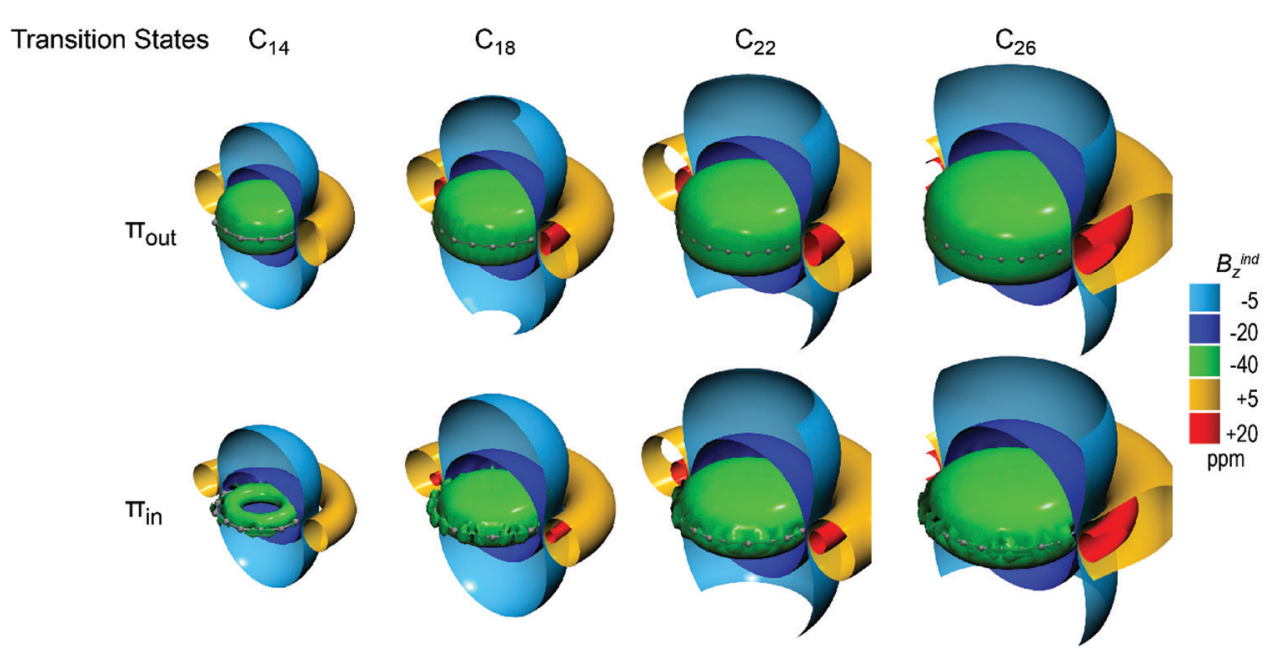

Fig. 6 Isosurfaces of $\pi_{\text {out }}$ (top) and $\pi_{\text {in }}$ (bottom) contributions to $B_{z}^{\text {ind }}$ of cumulenic transition states of $D_{(n / 2) h} C_{4 n+2}(n=3-6)$ rings. 


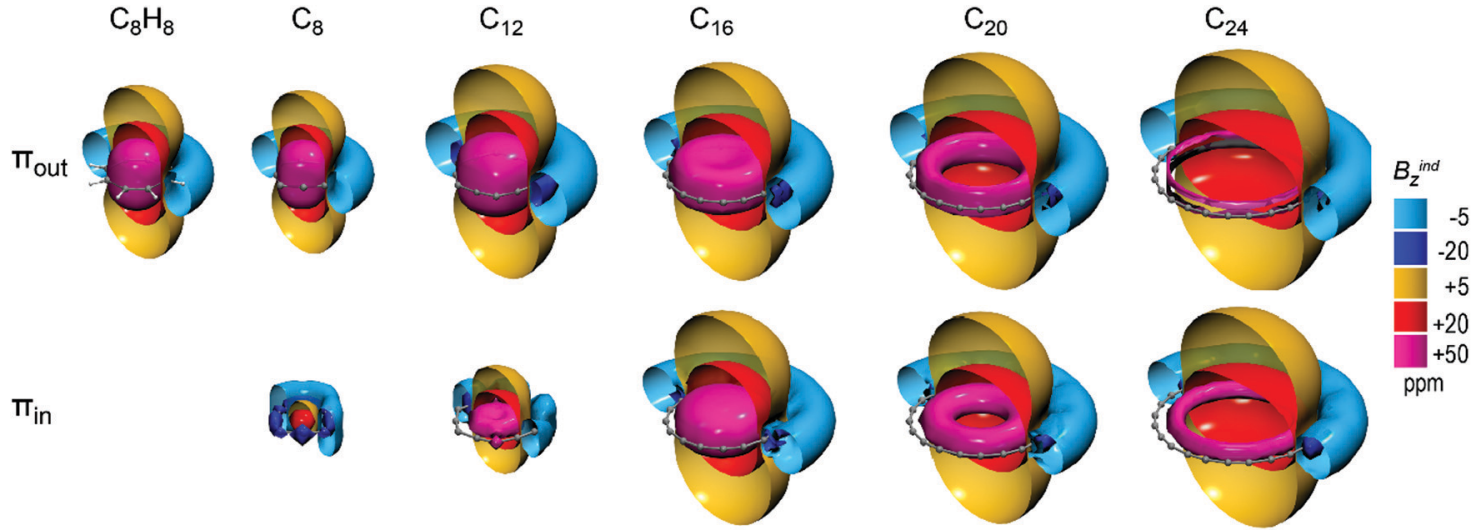

Fig. 7 Isosurfaces of $\pi_{\text {out }}$ (top) and $\pi_{\text {in }}$ (bottom) contributions to $B_{z}^{\text {ind }}$ of $C_{4 n}(n=2-6)$ rings and planar $\mathrm{C}_{8} \mathrm{H}_{8}$.

of lower orbitals, whereas in aromatic molecules the HOMOs induce a very weak paratropic or diatropic response that adjusts the overall diatropicity and tunes the aromaticity. In turn, the paratropic response of HOMOs originates from symmetry allowed rotational excitations to unoccupied orbitals and its magnitude depends on the energy gap and the overlap of interacting orbitals. ${ }^{43,44,46,58-60}$

In $\mathrm{C}_{4 n}$ rings, the HOMO and LUMO of both out-of-plane and in-plane orientations are non-degenerate MOs with the same number of $n$ nodal planes and the same symmetry. Hence the symmetry allowed $\mathrm{HOMO}_{\text {out/in }} \rightarrow \mathrm{LUMO}_{\text {out/in }}$ excitations, rotating the HOMOs by an angle of $2 \pi / 4 n$, to lead to an optimum overlap producing maximum paratropic response. For example, in $\mathrm{C}_{16}$ the rotational excitations of $\mathrm{HOMO}_{\text {out }}$ and $\mathrm{HOMO}_{\text {in }}$ with 4 nodal planes rotated by $22.5^{\circ}$ lead to a perfect overlap with $\mathrm{LUMO}_{\text {out }}$ and $\mathrm{LUMO}_{\text {in }}$ contributing with +63.3 and $+64.4 \mathrm{ppm}$, respectively, to $\mathrm{NICS}_{z z}$ (Fig. 8b) and induce very strong long range deshielding cones (Fig. 8a) that

a)

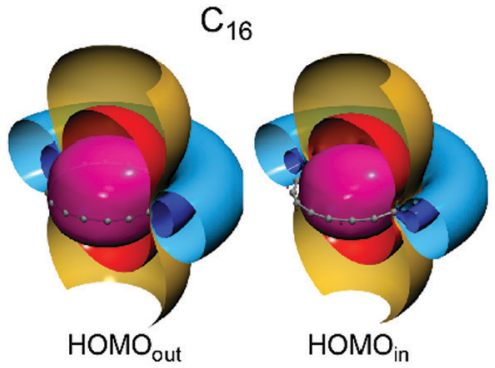

$\mathrm{C}_{16}$

$\mathrm{C}_{18}$

$\mathrm{C}_{18}$ TS

b)
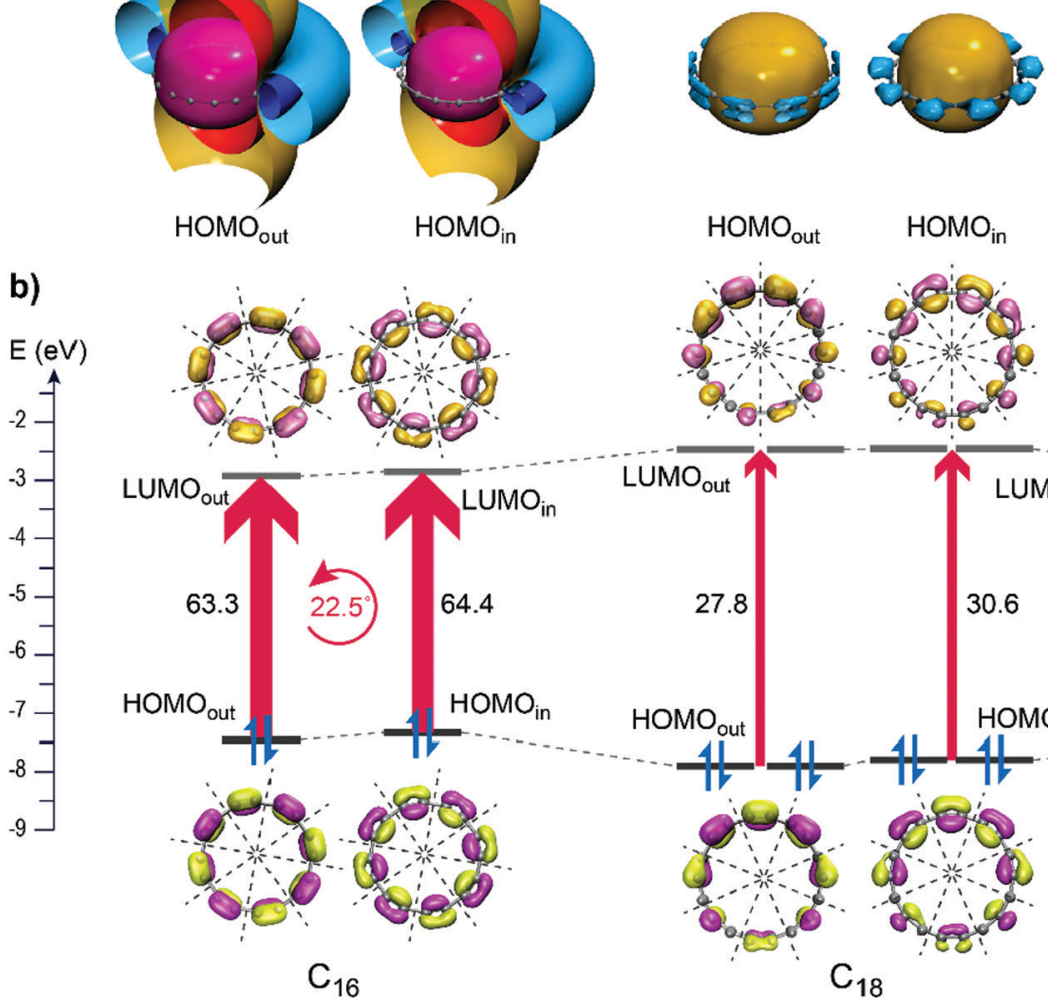

$\mathrm{HOMO}_{\text {out }} \quad \mathrm{HOMO}_{\text {in }}$
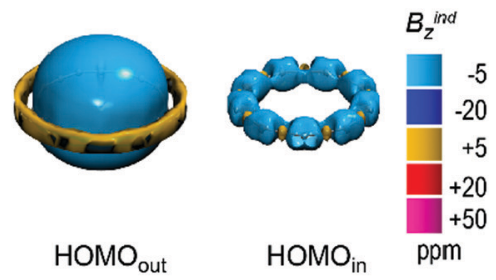

$\mathrm{HOMO}_{\text {out }}$
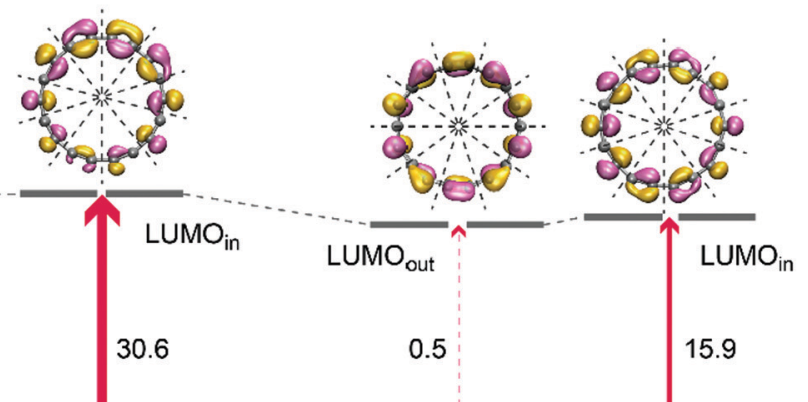
dominate on the overall magnetic response. As the HOMOLUMO gaps remain practically unchanged throughout the $\mathrm{C}_{4 n}$ series (Table S4, ESI $\dagger$ ), the decline in paratropicity originates from a weakening paratropic response of HOMOs and is attributed to the decrease in rotational overlap as the number of nodal planes increases.

In $\mathrm{C}_{4 n+2}$ rings, both $\mathrm{HOMO}_{\text {out }}$ and $\mathrm{HOMO}_{\text {in }}$ are doubly degenerate with $n$ nodal planes, whereas the LUMOs are doubly degenerate with $n+1$ nodal planes. In a perfect symmetry of $D_{n \mathrm{~h}}$ point group a HOMO $\rightarrow$ LUMO rotational excitation would be symmetry forbidden, but in $D_{(n / 2) \text { h }}$ and $C_{(n / 2) \text { h }}$ point groups such excitations are symmetry allowed. However due to a different number of nodal planes the overlap is small, inducing a weak magnetic response that depends on the geometrical characteristics of the ring. For example in $C_{9 \mathrm{~h}} \mathrm{C}_{\mathbf{1 8}}$, the frontier orbitals are distorted due to significant BLA and the HOMO $\rightarrow$ LUMO excitations display a small overlap inducing weak paratropic response (Fig. 8a) that diminishes the diatropicity of the lower energy orbitals, contributing with +27.8 and $+30.6 \mathrm{ppm}$ to NICS $_{z z}$ of $\pi_{\text {out }}$ and $\pi_{\text {in }}$, respectively (Fig. 8b). In contrast, in $D_{9 \mathrm{~h}} \mathrm{C}_{18}$ the zero BLA leads to a negligible rotational overlap of $\mathrm{HOMO}_{\text {out }} \rightarrow \mathrm{LUMO}_{\text {out }}$ excitation, resulting in the diatropic response of $\mathrm{HOMO}_{\text {out }}$ that adds to the overall diatropicity. On the other hand, $\mathrm{HOMO}_{\text {in }} \rightarrow \mathrm{LUMO}_{\text {in }}$ excitations of $D_{9 \mathrm{~h}}$ $\mathrm{C}_{18}$ induce small paratropic contributions (+15.9 ppm) due to increased BAA that leads to a weaker diatropicity of $\pi_{\text {in }}$ with regard to $\pi_{\text {out }}$. The same holds for the ground states of smaller members $\mathrm{C}_{6}, \mathrm{C}_{10}$ and $\mathrm{C}_{14}$ with zero (or almost zero) BLA, which induce shielding cones from $\mathrm{HOMO}_{\text {out }}$ (Fig. S5, ESI $\dagger$ ) and display increased $\pi_{\text {out }}$ diatropicity. For $\mathrm{C}_{4 n+2}$ ground states the increment in HOMO nodal planes with the ring size results in a gradual increment in the small overlap and hence in the augmentation of HOMO paratropic contributions (Fig. S5, ESI $\dagger$ ), causing a gradual decrement in the overall diatropicity.

\section{Conclusions}

The dissection of the induced magnetic field to contributions from out-of-plane and in-plane $\pi$ orbitals revealed the dual aromatic and antiaromatic character of $\mathrm{C}_{4 n+2}$ and $\mathrm{C}_{4 n}$ rings, respectively, according to the magnetic criterion. The magnetic response induced from distinctive sets of electrons is sensitive to the bonding pattern and geometrical features, where polyynic $\mathrm{C}_{4 n+2}$ ground states display similar out-of-plane and in-plane magnetic responses and comparable diatropicity to annulenes, while the cumulenic transition states exhibit a very strong diatropic character and an augmented out-of-plane magnetic response with regard to the in-plane magnetic response. Bond length alteration is the prime factor that constrains both $\pi_{\text {out }}$ and $\pi_{\text {in }}$ magnetic responses, whereas the bond angle alteration affects secondarily only the $\pi_{\text {in }}$ magnetic response. A significant decline in magnetic response with an increment in the ring size is observed for both ground state aromatic and antiaromatic rings, which is attributed to decreasing paratropic contributions of HOMOs originating from rotational excitations.

\section{Conflicts of interest}

There are no conflicts to declare.

\section{References}

1 H. W. Kroto, J. R. Heath, S. C. O'Brien, R. F. Curl and R. E. Smalley, C60: Buckminsterfullerene, Nature, 1985, 318, 162-163.

2 K. S. Novoselov, Electric Field Effect in Atomically Thin Carbon Films, Science, 2004, 306, 666-669.

3 S. Iijima, Helical microtubules of graphitic carbon, Nature, 1991, 354, 56-58.

4 Y. Wang, P. Yang, L. Zheng, X. Shi and H. Zheng, Carbon nanomaterials with $\mathrm{sp}^{2}$ or/and $\mathrm{sp}$ hybridization in energy conversion and storage applications: A review, Energy Storage Mater., 2020, 26, 349-370.

5 M. F. L. De Volder, S. H. Tawfick, R. H. Baughman and A. J. Hart, Carbon nanotubes: Present and future commercial applications, Science, 2013, 339, 535-539.

6 L. Bacakova, J. Pajorova, M. Tomkova, R. Matejka, A. Broz, J. Stepanovska, S. Prazak, A. Skogberg, S. Siljander and P. Kallio, Applications of Nanocellulose/Nanocarbon Composites: Focus on Biotechnology and Medicine, Nanomaterials, 2020, 10, 196.

7 J. L. Blackburn, A. J. Ferguson, C. Cho and J. C. Grunlan, Adv. Mater., 2018, 30, 1704386.

8 B. Han, Y. L. Zhang, Q. D. Chen and H. B. Sun, Adv. Funct. Mater., 2018, 28, 1802235.

9 N. Panwar, A. M. Soehartono, K. K. Chan, S. Zeng, G. Xu, J. Qu, P. Coquet, K. T. Yong and X. Chen, Chem. Rev., 2019, 119, 9559-9656.

10 A. Rajkamal and R. Thapa, Adv. Mater. Technol., 2019, 4, 1900307.

11 Y. Jiao, A. Du, M. Hankel and S. C. Smith, Modelling carbon membranes for gas and isotope separation, Phys. Chem. Chem. Phys., 2013, 15(14), 4832-4843.

12 P. Avouris, M. Radosavljević and S. Wind, Carbon Nanotube Electronics and Optoelectronics, in Applied Physics of Carbon Nanotubes, ed. S. V. Rotkin and S. Subramoney, NanoScience and Technology, Springer, Berlin, Heidelberg, 2005.

13 R. H. Baughman, H. Eckhardt and M. Kertesz, Structureproperty predictions for new planar forms of carbon: Layered phases containing $\mathrm{sp}^{2}$ and sp atoms, J. Chem. Phys., 1987, 87, 6687-6699.

14 F. Diederich, Y. Rubin, C. B. Knobler, R. L. Whetten, K. E. Schriver, K. N. Houk and Y. Li, All-Carbon Molecules: Evidence for the Generation of Cyclo[18]carbon from a Stable Organic Precursor, Science, 1989, 245, 1088-1090.

15 X. Gao, H. Liu, D. Wang and J. Zhang, Graphdiyne: synthesis, properties, and applications, Chem. Soc. Rev., 2019, 48, 908-936. 
16 C. Xie, N. Wang, X. Li, G. Xu and C. Huang, Research on the Preparation of Graphdiyne and Its Derivatives, Chem. - Eur. J, 2020, 26, 569-583.

17 R. Sakamoto, N. Fukui, H. Maeda, R. Matsuoka, R. Toyoda and H. Nishihara, The Accelerating World of Graphdiynes, Adv. Mater., 2019, 31, 1804211.

18 K. Kaiser, L. M. Scriven, F. Schulz, P. Gawel, L. Gross and H. L. Anderson, An sp-hybridized molecular carbon allotrope, cyclo[18]carbon, Science, 2019, 365, 1299-1301.

19 P. W. Fowler, N. Mizoguchi, D. E. Bean and R. W. A. Havenith, Double Aromaticity and Ring Currents in All-Carbon Rings, Chem. - Eur. J., 2009, 15, 6964-6972.

20 K. Remya and C. H. Suresh, Carbon rings: a DFT study on geometry, aromaticity, intermolecular carbon-carbon interactions and stability, RSC Adv., 2016, 6, 44261-44271.

21 C. Neiss, E. Trushin and A. Görling, The Nature of One-Dimensional Carbon: Polyynic versus Cumulenic, ChemPhysChem, 2014, 15, 2497-2502.

22 M. Feyereisen, M. Gutowski, J. Simons and J. Almlöf, Relative stabilities of fullerene, cumulene, and polyacetylene structures for Cn: $N=18-60, \mathrm{~J}$. Chem. Phys., 1992, 96, 2926-2932.

23 V. Parasuk, J. Almlof and M. W. Feyereisen, The [18] allcarbon molecule: cumulene or polyacetylene?, J. Am. Chem. Soc., 1991, 113, 1049-1050.

24 D. A. Plattner and K. N. Houk, C18 Is a Polyyne, J. Am. Chem. Soc., 1995, 117, 4405-4406.

25 T. Torelli and L. Mitas, Electron Correlation in C4N + 2 Carbon Rings: Aromatic versus Dimerized Structures, Phys. Rev. Lett., 2000, 85, 1702-1705.

26 S. Arulmozhiraja and T. Ohno, CCSD calculations on C14, C18, and C22 carbon clusters, J. Chem. Phys., 2008, 128, 114301.

27 A. J. Stasyuk, O. A. Stasyuk, M. Solà and A. A. Voityuk, Cyclo[18]carbon: the smallest all-carbon electron acceptor, Chem. Commun., 2020, 56, 352-355.

28 G. V. Baryshnikov, R. R. Valiev, A. V. Kuklin, D. Sundholm and H. Ågren, Cyclo[18]carbon: Insight into Electronic Structure, Aromaticity, and Surface Coupling, J. Phys. Chem. Lett., 2019, 10, 6701-6705.

29 É. Brémond, Á. J. Pérez-Jiménez, C. Adamo and J. C. SanchoGarcía, Sp-hybridized carbon allotrope molecular structures: An ongoing challenge for density-functional approximations, J. Chem. Phys., 2019, 151, 211104.

30 A. Nandi, E. Solel and S. Kozuch, Carbon Tunneling in the Automerization of Cyclo[18]carbon, Chem. - Eur. J., 2020, 26, 625-628.

31 R. Hoffmann, Chemical Abstracts Service: Cumulenes, polyenes, polyacetylenes and cn, Tetrahedron, 1966, 22, 521-538.

32 M. D. Wodrich, C. Corminboeuf, S. S. Park and P. Von Ragué Schleyer, Double aromaticity in monocyclic carbon, boron, and borocarbon rings based on magnetic criteria, Chem. - Eur. J., 2007, 13, 4582-4593.

33 R. Gershoni-Poranne and A. Stanger, Magnetic criteria of aromaticity, Chem. Soc. Rev., 2015, 44, 6597-6615.

34 M. K. Cyrański, Energetic Aspects of Cyclic Pi-Electron Delocalization: Evaluation of the Methods of Estimating
Aromatic Stabilization Energies, Chem. Rev., 2005, 105, 3773-3811.

35 J. Poater, M. Duran, M. Solà and B. Silvi, Theoretical Evaluation of Electron Delocalization in Aromatic Molecules by Means of Atoms in Molecules (AIM) and Electron Localization Function (ELF) Topological Approaches, Chem. Rev., 2005, 105, 3911-3947.

36 D. W. Szczepanik, M. Solà, M. Andrzejak, B. Pawełek, J. Dominikowska, M. Kukułka, K. Dyduch, T. M. Krygowski and $\mathrm{H}$. Szatylowicz, The role of the long-range exchange corrections in the description of electron delocalization in aromatic species, J. Comput. Chem., 2017, 38, 1640-1654.

37 G. Merino, T. Heine and G. Seifert, The Induced Magnetic Field in Cyclic Molecules, Chem. - Eur. J., 2004, 10, 4367-4371.

38 R. Islas, T. Heine and G. Merino, The Induced Magnetic Field, Acc. Chem. Res., 2012, 45, 215-228.

39 R. Gershoni-Poranne and A. Stanger, The NICS- XY -Scan: Identification of Local and Global Ring Currents in MultiRing Systems, Chem. - Eur. J., 2014, 20, 5673-5688.

40 R. Gershoni-Poranne and A. Stanger, Magnetic criteria of aromaticity, Chem. Soc. Rev., 2015, 44, 6597-6615.

41 I. G. Cuesta, A. S. De Merás, S. Pelloni and P. Lazzeretti, Understanding the ring current effects on magnetic shielding of hydrogen and carbon nuclei in naphthalene and anthracene, J. Comput. Chem., 2009, 30, 551-564.

42 R. Benassi, P. Lazzeretti and F. Taddei, Magnetic criteria for aromaticity, J. Phys. Chem., 1975, 79, 848-851.

43 N. D. Charistos, A. G. Papadopoulos and M. P. Sigalas, Interpretation of Electron Delocalization in Benzene, Cyclobutadiene, and Borazine Based on Visualization of Individual Molecular Orbital Contributions to the Induced Magnetic Field, J. Phys. Chem. A, 2014, 118, 1113-1122.

44 N. D. Charistos, A. G. Papadopoulos, T. A. Nikopoulos, A. Muñoz-Castro and M. P. Sigalas, Canonical orbital contributions to the magnetic fields induced by global and local diatropic and paratropic ring currents, J. Comput. Chem., 2017, 38, 2594-2604.

45 N. D. Charistos and A. Muñoz-Castro, Double aromaticity of the B 40 fullerene: induced magnetic field analysis of $\pi$ and $\sigma$ delocalization in the boron cavernous structure, Phys. Chem. Chem. Phys., 2019, 21, 20232-20238.

46 N. D. Charistos, A. Muñoz-Castro and M. P. Sigalas, The pseudo- $\pi$ model of the induced magnetic field: fast and accurate visualization of shielding and deshielding cones in planar conjugated hydrocarbons and spherical fullerenes, Phys. Chem. Chem. Phys., 2019, 21, 6150-6159.

47 J. Da Chai and M. Head-Gordon, Long-range corrected hybrid density functionals with damped atom-atom dispersion corrections, Phys. Chem. Chem. Phys., 2008, 10, 6615-6620.

48 A. Ehnbom, M. B. Hall and J. A. Gladysz, Origin of Shielding and Deshielding Effects in NMR Spectra of Organic Conjugated Polyynes, Org. Lett., 2019, 21, 753-757.

49 M. J. Frisch, G. W. Trucks, H. B. Schlegel, G. E. Scuseria, M. A. Robb, J. R. Cheeseman, G. Scalmani, V. Barone, G. A. Petersson, H. Nakatsuji, X. Li, M. Caricato, 
A. Marenich, J. Bloino, B. G. Janesko, R. Gomperts, B. Mennucci, H. P. Hratchian, J. V. Ortiz, A. F. Izmaylov, J. L. Sonnenberg, D. Williams-Young, F. Ding, F. Lipparini, F. Egidi, J. Goings, B. Peng, A. Petrone, T. Henderson, D. Ranasinghe, V. G. Zakrzewski, J. Gao, N. Rega, G. Zheng, W. Liang, M. Hada, M. Ehara, K. Toyota, R. Fukuda, J. Hasegawa, M. Ishida, T. Nakajima, Y. Honda, O. Kitao, H. Nakai, T. Vreven, K. Throssell, J. A. Montgomery, Jr., J. E. Peralta, F. Ogliaro, M. Bearpark, J. J. Heyd, E. Brothers, K. N. Kudin, V. N. Staroverov, T. Keith, R. Kobayashi, J. Normand, K. Raghavachari, A. Rendell, J. C. Burant, S. S. Iyengar, J. Tomasi, M. Cossi, J. M. Millam, M. Klene, C. Adamo, R. Cammi, J. W. Ochterski, R. L. Martin, K. Morokuma, O. Farkas, J. B. Foresman and D. J. Fox, Gaussian 09, Gaussian, Inc., Wallingford CT, 2016.

50 G. te Velde, F. M. Bickelhaupt, E. J. Baerends, C. Fonseca Guerra, S. J. A. van Gisbergen, J. G. Snijders and T. Ziegler, Chemistry with ADF, J. Comput. Chem., 2001, 22, 931-967.

51 ADF, SCM, Theoretical Chemistry, Vrije Universiteit, Amsterdam, The Netherlands, 2019, http://www.scm.com.

52 A. D. Becke, A new mixing of Hartree-Fock and local densityfunctional theories, J. Chem. Phys., 1993, 98, 1372-1377.

53 J. P. Perdew, K. Burke and M. Ernzerhof, Generalized gradient approximation made simple, Phys. Rev. Lett., 1996, 77, 3865-3868.
54 G. Schreckenbach and T. Ziegler, Calculation of NMR Shielding Tensors Using Gauge-Including Atomic Orbitals and Modern Density Functional Theory, J. Phys. Chem., 1995, 99, 606-611.

55 N. Charistos, T. Nikopoulos and M. Sigalas, MIMAF (Molecular Induced MAgnetic Fields) code, Laboratory of Quantum and Computational Chemistry, Department of Chemistry, Aristotle University of Thessaloniki, Thessaloniki, 2016.

56 W. Humphrey, A. Dalke and K. Schulten, VMD: Visual molecular dynamics, J. Mol. Graphics, 1996, 14, 33-38.

57 F. Pichierri, Boron-nitrogen analogues of cyclo[18]carbon, Chem. Phys. Lett., 2020, 738, 136860.

58 C. Corminboeuf, R. B. King and P. von R. Schleyer, Implications of Molecular Orbital Symmetries and Energies for the Electron Delocalization of Inorganic Clusters, ChemPhysChem, 2007, 8, 391-398.

59 I. Pérez-Juste, M. Mandado and L. Carballeira, Contributions from orbital-orbital interactions to nucleus-independent chemical shifts and their relation with aromaticity or antiaromaticity of conjugated molecules, Chem. Phys. Lett., 2010, 491, 224-229.

60 C. M. Widdifield and R. W. Schurko, Understanding chemical shielding tensors using group theory, MO analysis, and modern density-functional theory, Concepts Magn. Reson. Part A, 2009, 34A, 91-123. 